\title{
Aplicação dos modelos de Langmuir e Freundlich no estudo da casca de banana como bioadsorvente de cobre (II) em meio aquoso
}

\author{
Application of Langmuir and Freundlich \\ models in the study of banana peel as \\ bioadsorbent of copper (II) in aqueous \\ medium
}

Emerson Leandro-Silva ${ }^{1}$, Angelo Ricardo Favaro Pipi ${ }^{2}$, Aroldo Geraldo Magdalena ${ }^{3}$, Marina Piacenti-Silva ${ }^{4}$

\footnotetext{
${ }^{1}$ Pós-Graduação em Engenharia Civil e Ambiental - Universidade Estadual Paulista, UNESP, CEP: 17033-360, Bauru, SP, Brasil.

${ }^{2}$ National Centre For Sensor Research, School of Chemical Sciences, Dublin City University, Dublin 9, Ireland.

${ }^{3}$ Departamento de Química/Universidade Estadual Paulista - UNESP, Faculdade de Ciências, CEP: 17033-360, Bauru, SP, Brasil.

${ }^{4}$ Departamento de Física/Universidade Estadual Paulista - UNESP, Faculdade de Ciências, CEP: 17033-360/Bauru, SP, Brasil.

e-mail: emerson.silva@unesp.br, angelfav@gmail.com, aroldo.magdalena@unesp.br, marina.piacenti@unesp.br
}

\section{RESUMO}

A contaminação de água por metais pesados ainda é um grande risco de poluição. Diante deste cenário, processos adsortivos e diversos materiais adsorventes têm sido empregados para descontaminação. Dentre os materiais sintéticos e bioadsorventes empregados, os sintéticos são eficientes na remoção de metais em solução aquosa, contudo agregam alto custo comparados aos bioadsorventes. Os bioadsorventes são em geral provenientes de biomassa agroindustrial e vêm se destacando por serem abundantes, renováveis e com custo acessível para remediação de efluentes com metais pesados na forma ionizada. Dentre os bioadsorventes, a casca da banana reúne grupos funcionais capazes de adsorver íons de metais pesados em meio aquoso. Dessa forma, este trabalho descreve o método de preparação e caraterização da farinha da casca de banana (FCB) através das técnicas de Microscopia Eletrônica de Varredura (MEV), Espectroscopia Dispersiva em Energia de Raios X e Espectroscopia na Região do Infravermelho bem como estudos da capacidade adsortiva desse bioadsorvente para o íon $\mathrm{Cu}$ (II) em soluções aquosas. Os estudos de adsorção foram realizados através da avaliação das isotermas experimentais de adsorção em função do tempo de contato, variações do pH, temperatura e concentração de íon $\mathrm{Cu}$ (II) na solução pela técnica de batelada. As isotermas experimentais foram submetidas aos modelos matemáticos de isotermas de Langmuir e Freundlich. A capacidade máxima de adsorção alcançada pela FCB foi $15,1 \mathrm{mg}$ de Cu (II) por grama de FCB, pH ajustado em 5 e tempo de saturação bastante rápido, em torno de 10 minutos. A isoterma de Langmuir foi o modelo que melhor se ajustou ao processo adsortivo de cobre (II) pela FCB e o preenchimento dos sítios ativos foi na faixa de 98,7\%.

Palavras-chave: Bioadsorvente. Musa spp. Metal pesado. Biorremediação.

\section{ABSTRACT}

Contamination of water by heavy metals is still a major risk of pollution. Considering this scenario, adsorptive processes and various adsorbent materials have been used for decontamination. Among the synthetic materials and bioadsorbents used, the synthetics are efficient in the removal of metals in aqueous solution, however they add a high cost compared to bioadsorbents. The bioadsorbents are generally derived from agroindustrial biomass and have been noted for being abundant, renewable and cost-effective for the remediation of effluents with heavy metals in the ionized form. Among the bioadsorbents, the banana peel contains 
functional groups capable of adsorbing heavy metal ions in aqueous medium. Thus, this work describes the method of preparation and characterization of the banana peel flour through the techniques of Scanning Electron Microscopy (SEM), Dispersive Energy of x-ray (EDX) and Fourier Transform Infrared Spectroscopy (FT-IR), as well as studies of the adsorptive capacity of this bioadsorbent for the $\mathrm{Cu}$ (II) ion in aqueous solutions. The adsorption studies were carried out by evaluating experimental adsorption isotherms as a function of contact time, $\mathrm{pH}$, temperature and $\mathrm{Cu}$ (II) ion concentration in the solution by batch technique. The experimental isotherms were submitted to the mathematical models of Langmuir and Freundlich isotherms. The maximum adsorption capacity achieved by the FCB was $15.1 \mathrm{mg}$ of $\mathrm{Cu}$ (II) per gram of FCB, adjusted $\mathrm{pH}$ at 5 and very fast saturation time, around 10 minutes. The Langmuir isotherm was the model that best fitted the copper (II) adsorptive process by FCB and the filling of the active sites was around 98.7\%.

Keywords: Bioadsorbent. Musa spp. Heavy metal. Bioremediation.

\section{INTRODUÇÃO}

A preocupação com o meio ambiente e seus recursos naturais vem ganhando grandes proporções devido à enorme quantidade de resíduos gerada pelo homem, assim como a elevada gama de produtos industrializados proporcionam um volume significativo de espécies químicas nocivas à saúde. Os metais pesados, que estão presentes na maioria dos efluentes industriais, vêm ganhando uma atenção especial pela toxicidade à saúde humana e por acarretar enorme desequilíbrio ambiental [1]. Esses resíduos se encontram na sua forma ionizada, em meio aquoso, e necessitam ser removidos antes que sejam despejados em corpos d'água e/ou solos, pois são compostos extremamente recalcitrantes, não degradando facilmente na natureza pela ação do tempo [2]. Nesse sentido, estudos buscam alternativas para tratar os efluentes com metais que sejam eficazes, renováveis e menos onerosas [3].

Uma alternativa muito eficaz no tratamento de efluentes com resíduos metálicos é a técnica de adsorção [4]. O carvão ativado é o adsorvente mais utilizado pela indústria devido sua versatilidade e eficácia no processo adsortivo para remediação de metais em efluentes [5,6]. Entretanto, o carvão ativado demanda um alto custo quando comparado aos bioadsorventes [1].

No sentido de reduzir os resíduos metálicos gerados nas atividades industriais e se adequar aos padrões rigorosos da legislação, a bioadsorção se torna uma alternativa economicamente viável por ser renovável e de baixo custo [7]. O mecanismo da bioadsorção é realizado pelas interaç̃os químicas ou eletrostáticas entre metais ionizados e alguns grupos funcionais da biomassa vegetal [8].

Dentre os bioadsorventes, destaca-se a casca da banana, por ser um fruto consumido com abundância em todo Brasil, cuja casca normalmente é descartada [9]. A casca de banana possui inúmeros sítios ativos responsáveis pela adsorção de metais, constituídos por vários grupos do tipo carbonilas, fenóis, carboxilas, hidroxilas e aminas que são provenientes de grupos funcionais como a celulose, holocelulose, lignina, ácido péctico, ácidos orgânicos e proteínas $[2,5,7,10]$. Portanto, possuindo estas características, torna-se viável a utilização da casca de banana como bioadsorvente por ser abundante, natural, renovável, com custo acessível e por conter propriedades adsortivas para metais ionizados em meio aquoso. Em meio aos diversos metais que podem ser bioadsorvidos pela casca de banana, destaca-se o Cu (II), por ser nocivo à saúde humana quando em altas concentração e está presente em muitos processos industriais [11]. Somando-se a isso, efluentes com altas concentrações deste metal devem ser tratados para descarte em corpos receptores classe II pois a concentração máxima de $\mathrm{Cu}$ (II) permitida no Brasil para efluentes industriais e/ou esgotos é de 1,00 $\mathrm{mg} \mathrm{L} \mathrm{L}^{-1}[12]$.

Diante deste cenário, o objetivo principal deste trabalho foi estudar a eficiência adsortiva de Cobre (II) usando a casca de banana como bioadsorvente, além de realizar a caracterização estrutural da casca de banana e ajustar os dados experimentais do estudo adsortivo aos modelos das isotermas de Langmuir e Freundlich.

\section{MATERIAIS E MÉTODOS}

\subsection{Pré-tratamento da casca de banana}

Neste trabalho foi utilizada a casca de banana nanica (Musa spp). As cascas coletadas (aproximadamente 2 $\mathrm{kg}$ ) foram secas ao sol por 72 horas, posteriormente cortadas em aproximadamente $5 \mathrm{~cm}$ de comprimento e lavadas com água deionizada. Em seguida, o material foi seco em estufa com circulação de ar, entre 50 e $60^{\circ} \mathrm{C}$ pelo período de 24 horas. O material obtido foi triturado em liquidificador industrial portátil (Siemens) e peneirado na granulometria entre 25-100 mesh. Nesta etapa, obteve-se uma farinha de casca de banana (FCB) que, em seguida, foi dispersa em solução de $\mathrm{HCl} 0,05 \mathrm{~mol} \mathrm{~L}^{-1}$ e mantida em repouso por 10 minutos 
em temperatura ambiente. Logo, o material foi lavado abundantemente com água deionizada, filtrado e seco em uma estufa por 72 horas com temperatura controlada entre 50 e $60^{\circ} \mathrm{C} \mathrm{[5].}$

\subsection{Caracterização do adsorvente}

O adsorvente FCB foi submetido ao estudo de caracterização empregando as seguintes técnicas: Microscopia Eletrônica de Varredura (MEV), obtidas no microscópio FE-SEM, JEOL 7500F, feixe de 10 kV e magnificação de 1000 vezes; Espectroscopia Dispersiva em Energia de Raios X (EDX) com energia de excitação de 10 $\mathrm{kV}$; Espetroscopia na região do infravermelho com método de refletância total atenuada (FT-IR ATR Vertex 70 - Bruker), com faixa de varredura entre 4000 e $400 \mathrm{~cm}^{-1}$ (32 scans e resolução de $4 \mathrm{~cm}^{-1}$ ) com cristal de diamante como suporte. As análises de MEV e EDX foram realizadas antes e após o tratamento químico da FCB, e também após a adsorção. Além disso, o adsorvente foi submetido ao processo de desidratação antes de todas as técnicas de caracterização e, posteriormente, fixada em fita de carbono no stub e metalizada com banho de ouro durante dois minutos para as análises de MEV e EDX, já para a aquisição dos dados do FT-IR a amostra seca foi colocada sobre o cristal do ATR e analisada.

\subsection{Estudos de adsorção}

Para os estudos adsortivos uma solução estoque de cobre foi preparada na concentração de $636 \mathrm{mg} \mathrm{L}^{-1}$, e suas respectivas diluições em 6,4;64; 191 e $572 \mathrm{mg} \mathrm{L}^{-1}$. Todos estudos de adsorção foram realizados em bateladas com volume de 0,025 L, 0,1 g de FCB, agitação média de $200 \mathrm{rpm}$. Para avaliar a capacidade adsortiva da FCB foram realizados os seguintes estudos:

a) Estudo Cinético de Adsorção com a finalidade de determinar o tempo necessário para a saturação do bioadsorvente FCB pelo íon cobre, buscando conhecer o tempo de equilíbrio adsortivo. Utilizado uma solução de concentração inicial $191 \mathrm{mg} \mathrm{L}^{-1}$ de $\mathrm{Cu}$ (II) e tempo de contato de 5, 10, 20, 30 e 60 minutos, pH fixado em 5,0 e temperatura ambiente $26^{\circ} \mathrm{C}( \pm 2)$;

b) Estudo da Influência do $\mathrm{pH}$ para determinar o pH da solução de cobre em que ocorre a máxima adsorção pela FCB. Para isso, utilizou-se uma solução de concentração inicial $191 \mathrm{mg} \mathrm{L}^{-1}$ de Cu (II) e pHs entre 3,0 e 7,0 monitorados por phmetro portátil digital (PhTek modelo PH-203). Para este estudo foi fixado o melhor tempo de contato obtido no estudo anterior e temperatura ambiente $26{ }^{\circ} \mathrm{C}( \pm 2)$. $\mathrm{O}$ controle do $\mathrm{pH}$ foi feito através de uma solução de $\mathrm{NaOH}$ preparada em duas concentrações $\left(0,04\right.$ e $\left.0,10 \mathrm{~mol} \mathrm{~L}^{-1}\right)$;

c) Estudo da Variação de Temperatura para avaliar a influência da temperatura no processo adsortivo da FCB. Desse modo, foram mantidos os melhores parâmetros anteriores de tempo e pH, e uma solução de concentração inicial $191 \mathrm{mg} \mathrm{L}^{-1}$ de $\mathrm{Cu}$ (II). As temperaturas trabalhadas foram $15,26,35$ e $45{ }^{\circ} \mathrm{C}$, as bateladas foram realizadas em equipamento de banho termostático (QUIMIS ${ }^{\circledR}$ ) com auxílio de béqueres encamisados;

d) Estudo da Capacidade Adsortiva da FCB com a variação da concentração de cobre na solução, determinante para avaliar a concentração de $\mathrm{Cu}$ (II) capaz de saturar os sítios ativos do bioadsorvente FCB. Para isso, foram mantidos os melhores parâmetros anteriores de tempo e $\mathrm{pH}$, e temperatura de $26^{\circ} \mathrm{C}$. As concentrações iniciais de $\mathrm{Cu}$ (II) nas soluções estudadas variaram em 6,4; 64; 191; 572 e $636 \mathrm{mg} \mathrm{L}^{-1}$, respectivamente.

Após as bateladas, todas as soluções foram filtradas com auxílio de bomba de vácuo, o sobrenadante coletado e a análise quantitativa dos íons cobre nas soluções foram realizadas por um espectrofotômetro UVVIS da marca JENWAY 7305 - MARCONI, com comprimento de onda em $530 \mathrm{~nm}$. Para realizar a leitura neste comprimento de onda, foram adicionadas 2 gotas de etileno-di-amina nas cubetas com a solução de análise, pois o etileno-di-amina quando em contato com íons cobre em solução forma um complexo de cor azulada. Através de uma curva de calibração, as concentrações de $\mathrm{Cu}$ nas soluções pré e pós adsorção foram determinadas.

\subsection{Capacidade adsortiva e modelos de adsorção}

A quantidade de cobre adsorvido pelo adsorvente FCB no equilíbrio $\left(q_{e}\right)$ foi calculada pela equação de balanço de massa (equação 1) [13]:

$$
q e=\left[\left(C_{i}-C_{e}\right) V\right] / m
$$

na qual, $C_{i} e C_{e}$ são as concentrações de cobre inicial e no estado de equilíbrio na fase líquida em $\mathrm{mg} \mathrm{L}^{-1}$, respectivamente, $V(\mathrm{~L})$ é o volume da solução e $m$ a massa $(\mathrm{g})$ do adsorvente. 
O percentual de remoção do cobre (\%) da solução foi calculado pela equação 2:

$$
\%=\left[\left(C_{i}-C_{e}\right) 100\right] / C_{i}
$$

Muitos modelos de isotermas foram propostos para descrever dados experimentais sobre os valores de $q_{e}$ versus $C_{e}$. Dentre os mais aplicados para fenômenos de adsorção líquido-sólido podem ser citados os modelos e as equações de Langmuir e Freundlich [13, 14, 15]. O comportamento adsortivo dos íons metálicos $\mathrm{Cu}$ (II) pelo adsorvente FCB foi estudado através da aplicação desses dois modelos.

O modelo de Langmuir é o mais utilizado para representar processos adsortivos e confere os pressupostos de que existe um número definido de sítios e possuem energia equivalente para apenas uma molécula a ser adsorvida, as moléculas adsorvidas não interagem entre si e a adsorção ocorre em uma monocamada, representado pela Equação 3, ferramenta utilizada neste trabalho para confecção da isoterma de Langmuir não linear [16].

$$
q e=\left(q_{\max } K L C_{e}\right) /\left[1+\left(K L C_{e}\right)\right]
$$

onde $q_{\max }$ é o número de sítios disponíveis no sólido ou capacidade máxima de adsorção ( $\mathrm{mg} \mathrm{g}^{-1}$ ) pelo adsorvente e $K L$ é a constante de interação adsorvato/adsorvente ou constante de afinidade de Langmuir $\left(\mathrm{L} \mathrm{mg}^{-1}\right)$. Ambas variáveis são facilmente extraídas da função linearizada da isoterma de Langmuir representada pela Equação 4 e pela curva $C_{e} / q_{e}$ como função de $C_{e}$. O coeficiente angular da reta resulta em $1 /\left(q_{\max }\right)$, enquanto o coeficiente linear resulta em $1 /\left(K L q_{\max }\right)[5,13]$.

$$
\left(C_{e} / q_{e}\right)=\left(1 / q_{\max }\right) C_{e}+1 /\left(K L q_{\max }\right)
$$

Dentre as características fundamentais da isoterma de Langmuir, o fator de separação ou parâmetro de equilíbrio $(R L)$, que corresponde ao grau de desenvolvimento do processo de adsorção, é essencial para prever se um processo adsortivo é favorável ou desfavorável [17]. O valor de $R L$ adimensional neste trabalho foi obtido através da Equação 5.

$$
R L=1 /\left[1+\left(K L * C_{e}\right)\right]
$$

na qual, ${ }^{*} C_{i}$ é a concentração inicial do íon Cu (II) mais alta. Para valores de $R L$ entre 0 e 1 temos uma adsorção favorável, pois o adsorvato tem maior afinidade com o adsorvente, para valores de $R L>1$, o adsorvato tem maior afinidade com fase líquida à sólida, uma adsorção desfavorável [5, 13, 17].

Já o modelo de Freundlich não exige que a cobertura das camadas de adsorvato sobre o adsorvente se aproxime de um valor constante, que corresponde à formação de monocamada, mesmo que ocorra um aumento de $C_{e}$. Além disso, o modelo pressupõe que o valor de $q_{e}$ pode continuar a aumentar, à medida que $C_{e}$ aumenta [13]. Contudo, os dados experimentais que apresentam concentrações elevados e valores de $\mathrm{Ce}$ muito altos não são bem ajustados à equação de Freundlich [18]. Este modelo de isoterma é representado pela Equação 6, ferramenta utilizada para confecção da isoterma de Freundlich não linear.

$$
q_{e}=K F C_{e}^{(1 / n)}
$$

em que $n$ é chamado de coeficiente Freundlich, $1 / n$ é a constante relacionada à heterogeneidade da superfície e $K F$ a constante de capacidade de adsorção de Freundlich [5,13]. Ambos coeficientes são facilmente extraídos da função linearizada da isoterma de Freudlich representada pela Equação 7 e pela curva $\log q_{e}$ versus $\log C_{e}$. O coeficiente angular da reta resulta em $1 / n$, enquanto o coeficiente linear resulta em $\log K F$ [19].

$$
\log q_{e}=(1 / n) \log C_{e}+\log K F
$$

Deste modo, uma adsorção favorável produz um valor de $n$ entre 1 e 10 . Sendo assim, quanto maior o valor de $n$ mais forte a interação entre o adsorvato e adsorvente, pois $1 / n$ é mínimo. Logo, se o valor $1 / n$ for igual a 1, indica que a adsorção é linear e as energias para todos os sítios de adsorção são idênticas. Quando o valor de $1 / n$ for maior que o $n$, ou seja, $n$ menor que 1 sugere que o adsorvente tem maior interação com o solvente e não com o adsorvato, gerando uma adsorção desfavorável [13].

\section{RESULTADOS}




\subsection{Pré-tratamento e caracterização do adsorvente}

A Figura 1 apresenta as imagens obtidas por MEV da FCB antes e após o tratamento ácido, bem como após o processo adsortivo dos íons cobre. Observou-se que antes do tratamento ácido (Figura 1a) este material bioadsorvente apresenta superfícies lisas e com o aspecto bastante poroso. O tratamento ácido deste bioadsorvente levou à modificação de sua morfologia superficial, (Figura 1b) provocando a formação de uma superfície menos lisa com aspecto ainda mais poroso, podendo assim, aumentar a capacidade de adsorção [20]. Este resultado era esperado, pois o pré tratamento da FCB com solução ácida e posterior lavagem com água deionizada promove a ruptura de estruturas cristalinas como lignina, holocelulose e celulose da biomassa [21], além de atribuir elevada reatividade às fibras devido a polarização [22], promovendo também a abertura de novos sítios ativos.

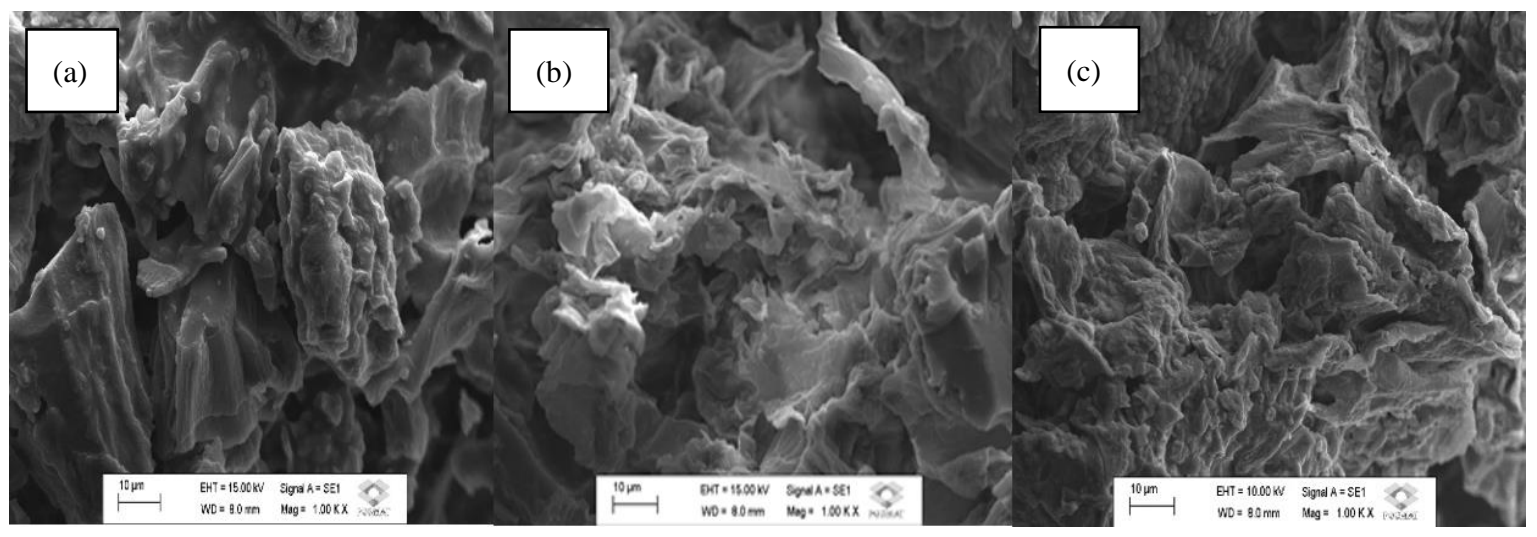

Figura 1: Imagens de Microscopia Eletrônica de varredura do bioadsorvente FCB (10 $\mu \mathrm{m})$ : (a) antes do tratamento ácido; (b) Após o tratamento ácido; (c) após o processo adsortivo do metal $\mathrm{Cu}$ (II).

Na fase de fruta, os inúmeros sítios de adsorção da casca da banana são preenchidos por íons metálicos provenientes do desenvolvimento natural da planta no meio ambiente, sendo assim, o tratamento com esta solução ácida promove a dessorção dos metais e a liberação desses sítios ocupados, facilitando a adsorção dos íons cobre e atribuindo maior eficiência. A lavagem abundante com água deionizada diminui a interferência eletrostática de compostos iônicos solúveis em água que possam intervir nas análises [23]. Após a adsorção (Figura 1c) é possível inferir que houve um preenchimento das superfícies que antes eram mais lisas, possivelmente devido a adsorção dos íons metálicos de cobre. Os dados obtidos na análise de composição EDX apresentados na Figura 2 corroboram com estas afirmações.
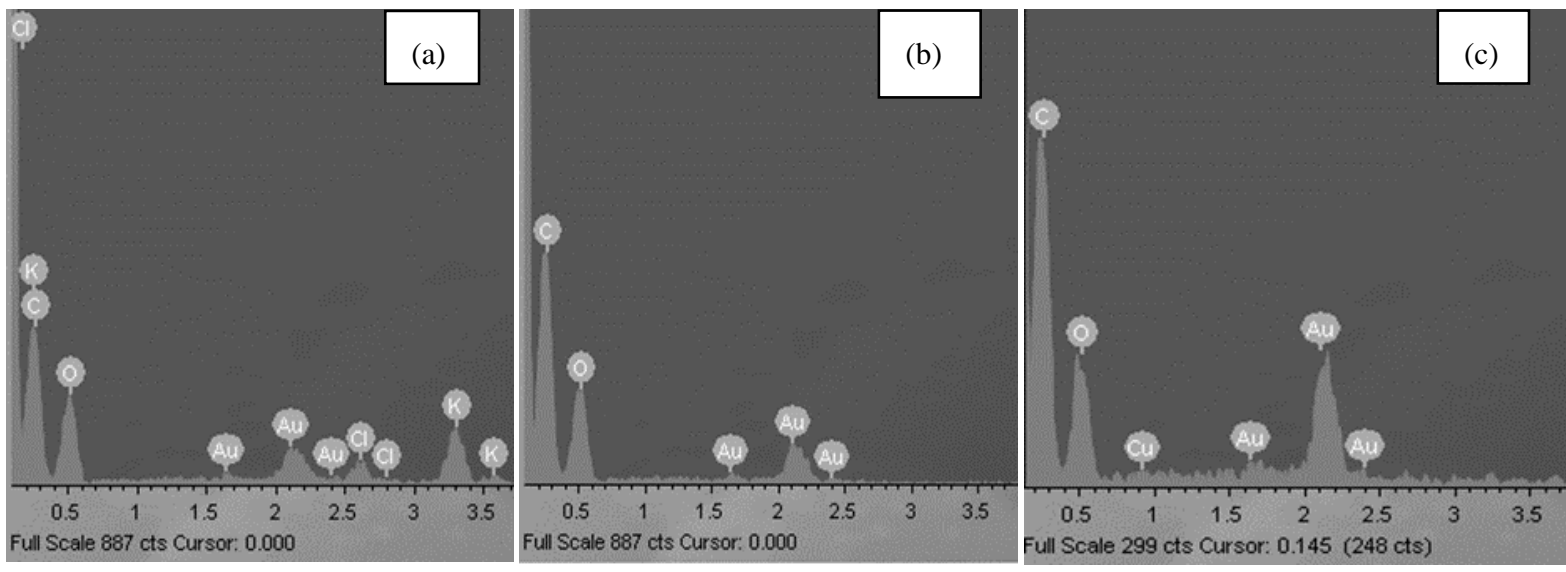

Figura 2: Análise de energia dispersiva de Raio X da FCB: (a) antes do tratamento ácido; (b) Após o tratamento ácido; (c) após o processo adsortivo do metal $\mathrm{Cu}$ (II).

Na Figura 2a, são apresentados os espectros dos componentes presentes na amostra, onde é possível observar diversos elementos como cloro $(\mathrm{Cl})$, carbono $(\mathrm{C})$, oxigênio $(\mathrm{O})$, potássio $(\mathrm{K})$ e o ouro ( $\mathrm{Au})$, este 
último proveniente da metalização da amostra, necessária para análise por MEV. Ao passo que a FCB foi submetida ao processo de tratamento ácido (Figura 2b) ocorre o desaparecimento dos elementos $\mathrm{K}$ e $\mathrm{Cl}$, confirmando a eficiência do pré-tratamento ácido na remoção de metais e outros compostos inorgânicos préexistentes na FCB e, paralelamente, a manutenção dos elementos $\mathrm{C}$ e $\mathrm{O}$ que evidencia a estrutura do bioadsorvente FCB que, por sua vez, se trata de um material lignocelulósico. Por fim, no espectro de EDX da FCB após a adsorção (Figura 2c) é possível observar a presença de íons cobre possivelmente proveniente do processo adsortivo, podendo inferir que este bioadsorvente é eficaz para remoção de cobre em solução aquosa.

Os espectros de FT-IR podem ser usados para identificar e explicar os grupos funcionais que interagem com ions metálicos por coordenação [20]. A Figura 3 apresenta o espectro de FT-IR do bioadsorvente FCB, que indica a presença de muitos grupos orgânicos possivelmente responsáveis pelas propriedades do bioadsorvente como: celulose, lignina, ácidos orgânicos, ésteres e proteínas [24].

A banda com pico intenso em $3290 \mathrm{~cm}^{-1}$ atribui-se ao estiramento vibracional axial dos grupos hidroxilas $(\mathrm{OH})$ proveniente da celulose [5,20]. As bandas espectrais na região de 2970 a $2840 \mathrm{~cm}^{-1}$ podem ser atribuídas aos grupos orgânicos alifáticos $\left(\mathrm{CH}-\mathrm{OH},-\mathrm{CH}\right.$ e $\left.-\mathrm{CH}_{2}\right)$ presentes na estrutura molecular da celulose [5,20]. A banda espectral em $1734 \mathrm{~cm}^{-1}$ pode ser atribuída a vibrações de deformação axial da carbonila $(\mathrm{C}=\mathrm{O})$ característico dos ácidos carboxílicos tais como o ácido péctico e/ou ácido cítrico ou até mesmo de ésteres presentes na casca de banana [25]. A banda intensa em $1610 \mathrm{~cm}^{-1}$ pode ser atribuída a vibração de estiramento do ânion (COO-) ou do grupo -CO- característicos dos ésteres ou éteres [5]. As bandas entre 1020 a $1300 \mathrm{~cm}^{-1}$ pertencem ao grupo orgânico CO [20].

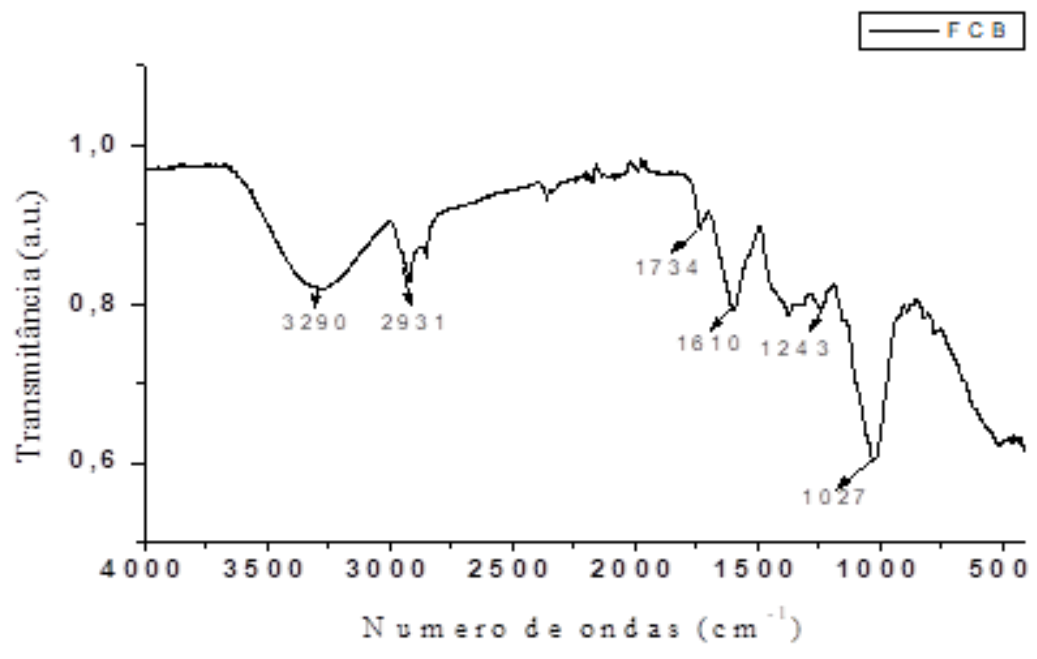

Figura 3: Espectros de FTIR do adsorvente FCB antes do tratamento ácido.

Portanto, a análise da FCB indicou que esse bioadsorvente contém grupos hidroxilas (celulose contém diferentes grupos $\mathrm{OH}$ ) e carboxilas que são capazes de reagir com íons de $\mathrm{Cu}$ (II) em meio aquoso. Portanto, a presença desses grupos funcionais torna a FCB um bioadsorvente promissor para remoção de íons metálicos em solução aquosa $[5,20]$

\subsection{Estudo das adsorções}

\subsubsection{Estudo cinético de adsorção}

A Figura 4 apresenta a quantidade de cobre adsorvido pelo adsorvente FCB no equilíbrio $\left(q_{e}\right)$ em função do tempo de adsorção. É possível observar que a adsorção dos íons cobre é relativamente rápida, pois com 5 minutos nota-se uma adsorção significativa e após 20 minutos o sistema entra em equilíbrio, onde possivelmente todos os sítios ativos do material bioadsorvente FCB foram preenchidos. 


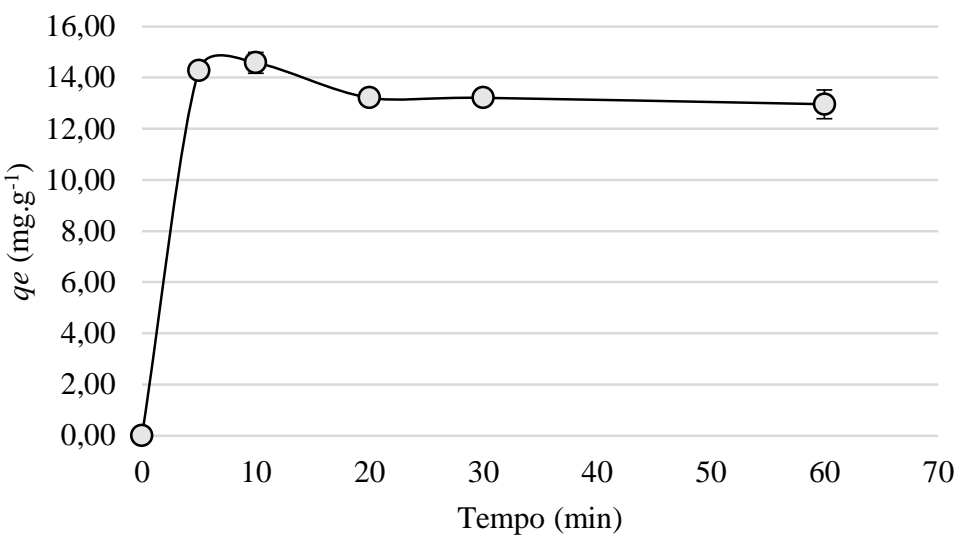

Figura 4: Quantidade de cobre adsorvido pelo adsorvente FCB no equilíbrio $\left(q_{e}\right)$ em função do tempo de adsorção.

De acordo com a Figura 4 nota-se que após 10 minutos há uma adsorção máxima, podendo ser utilizado também como tempo de equilíbrio de adsorção. A partir deste resultado, foi fixado em 10 minutos o tempo de contato empregado nas demais etapas.

\subsubsection{Estudo da influência do pH no processo adsortivo}

$\mathrm{O}$ pH exerce grande influência no processo de adsorção dos íons cobre pela FCB, conforme observado na Figura 5. Os resultados mostram que em pH 5 foi possível alcançar o máximo de adsorção e eficiência na remoção de íons cobre, resultado corroborado por Cruz [5]. Observa-se ainda que a capacidade de adsorção diminui com o decréscimo do $\mathrm{pH}$ da solução. Este resultado sugere um fenômeno de competição pelo sítio ativo do adsorvente entre os íons cobre e os prótons $\left(\mathrm{H}^{+}\right)$presente em solução, bem como a formação de possíveis complexos entre os metais, ânions e a água com cargas eletricamente neutras, ou ainda a tendência das cargas superficiais do adsorvente estarem carregadas positivamente por prótons proveniente do meio pelo declínio do $\mathrm{pH}[13,26]$.

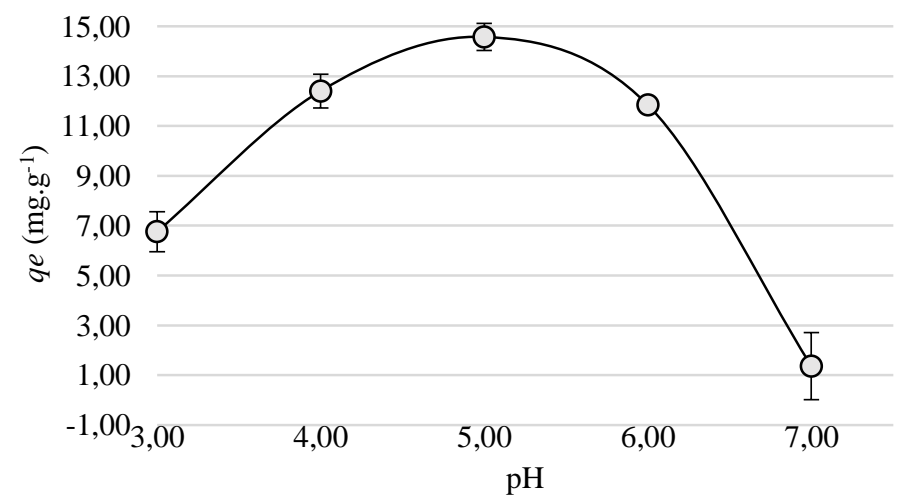

Figura 5: Quantidade de cobre adsorvido pelo adsorvente FCB no equilíbrio $\left(q_{e}\right)$ em função do $\mathrm{pH}$.

Para pHs superiores, a adsorção também sofre um declínio, que pode ser explicado pela diminuição dos íons metálicos disponíveis no meio, devido à possível formação de espécies do tipo $\mathrm{Cu}(\mathrm{OH})^{+}$. Em $\mathrm{pHs}$ acima de 6,5 foi evidenciada a formação de um precipitado, possivelmente hidróxido de cobre $\left(\mathrm{Cu}(\mathrm{OH})_{2}\right)$ devido à alta concentração da espécie $(\mathrm{OH})^{-}$no meio. De acordo com os resultados encontrados nesta etapa, fixou-se o pH 5 para os estudos seguintes.

\subsubsection{Estudo da variação de temperatura}

A Figura 6 ilustra a quantidade de cobre adsorvido pelo adsorvente FCB no equilíbrio $\left(q_{e}\right)$ em função da vari- 
ação da temperatura. Como observado na Figura 6, não houve uma variação significativa do processo adsortivo nas temperaturas 15 e $26^{\circ} \mathrm{C}$, contudo, ao passo que a temperatura foi aumentada houve um decréscimo da adsorção dos íons Cu (II). Este fenômeno não é incomum, pois devido ao aumento da temperatura sugere o favorecimento do mecanismo de dessorção, desse modo o processo adsortivo descreve uma interação entre o íon $\mathrm{Cu}$ (II) e o sítio do adsorvente FCB bastante reversível [17, 27]. Por outro lado, o estudo da variação da temperatura pode justificar a probabilidade de a adsorção ser física ou química e, normalmente, a adsorção física não ocorre em temperaturas elevadas, dessa maneira os resultados obtidos sugerem uma interação de natureza física entre o metal cobre e o material adsorvente [27, 28]. Logo, na temperatura de $26{ }^{\circ} \mathrm{C}$ existe uma forte interação adsorvato-adsorvente, pois nessa temperatura apresentou maior $q_{e}$.

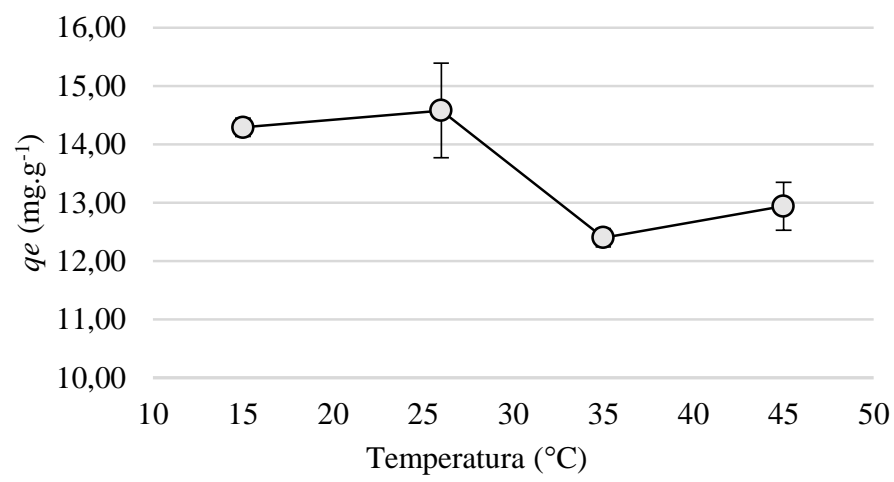

Figura 6: Quantidade de cobre adsorvido pelo adsorvente FCB no equilíbrio $\left(q_{e}\right)$ em função da temperatura.

\subsubsection{Estudo da Capacidade Adsortiva da FCB}

No estudo da variação da capacidade adsortiva do bioadsorvente FCB, as concentrações foram variadas de acordo com a metodologia descrita, o tempo do processo adsortivo foi de 10 minutos em $\mathrm{pH}$ 5, e a temperatura em $26{ }^{\circ} \mathrm{C}$ para viabilidade energética. Desta forma, foi obtida a isoterma da Figura 7a por meio da $q_{e} v e r-$ sus $C_{i}$ de íons Cu (II) e a curva da Figura 7b expressa o valor de saturação máxima através da relação $q_{e}$ versus $C_{e}$.

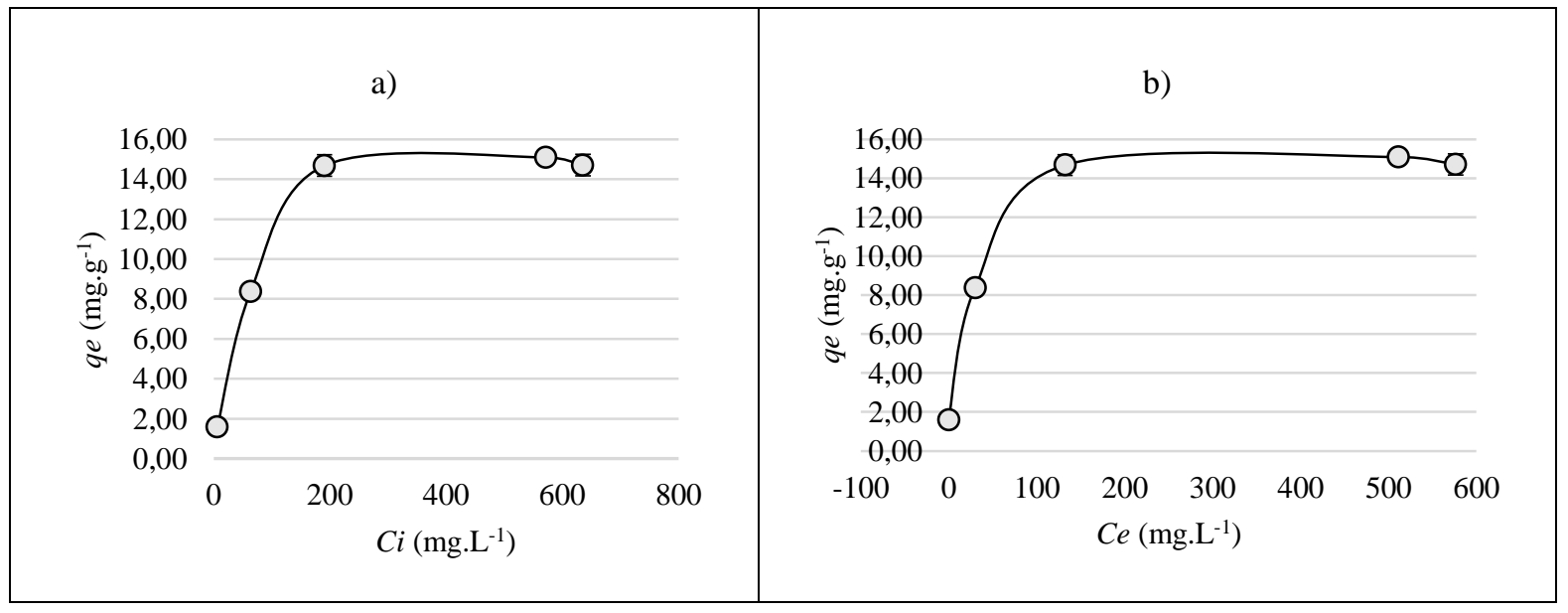

Figura 7: Quantidade de cobre adsorvido pelo adsorvente FCB no equilíbrio $\left(q_{e}\right)$ em função da (a) variação da concentração inicial empregando o bioadsorvente FCB e (b) variação da concentração final no equilíbrio após empregado o bioadsorvente FCB.

De acordo com as Figuras 7a e 7b, é possível observar que a saturação dos sítios ativos do material bioadsorvente FCB pelo íon cobre ocorre a partir da concentração próxima de $200 \mathrm{mg} \mathrm{L}^{-1}$. Nota-se ainda que o adsorvente admitiu uma concentração máxima de cobre em sua superfície, em torno de $15,1 \mathrm{mg} \mathrm{g}^{-1}$. Este equilíbrio pode ser justificado pelo preenchimento dos sítios ativos pelos íons de cobre através de interações físicas e/ou de coordenação entre os grupos funcionais e os cátions Cu (II) [5,13]. 


\subsubsection{Capacidade adsortiva e modelos de adsorção}

Os resultados experimentais foram ajustados para as isotermas de Langmuir e Freundlich. Desse modo, as curvas das equações linearizadas de Langmuir e Freundlich, de acordo com as equações 3 e 4, estão representadas nas Figuras 8a e 8b. A partir desses dados foram obtidos os parâmetros que indicam se a adsorção foi ou não favorável $[5,13]$.

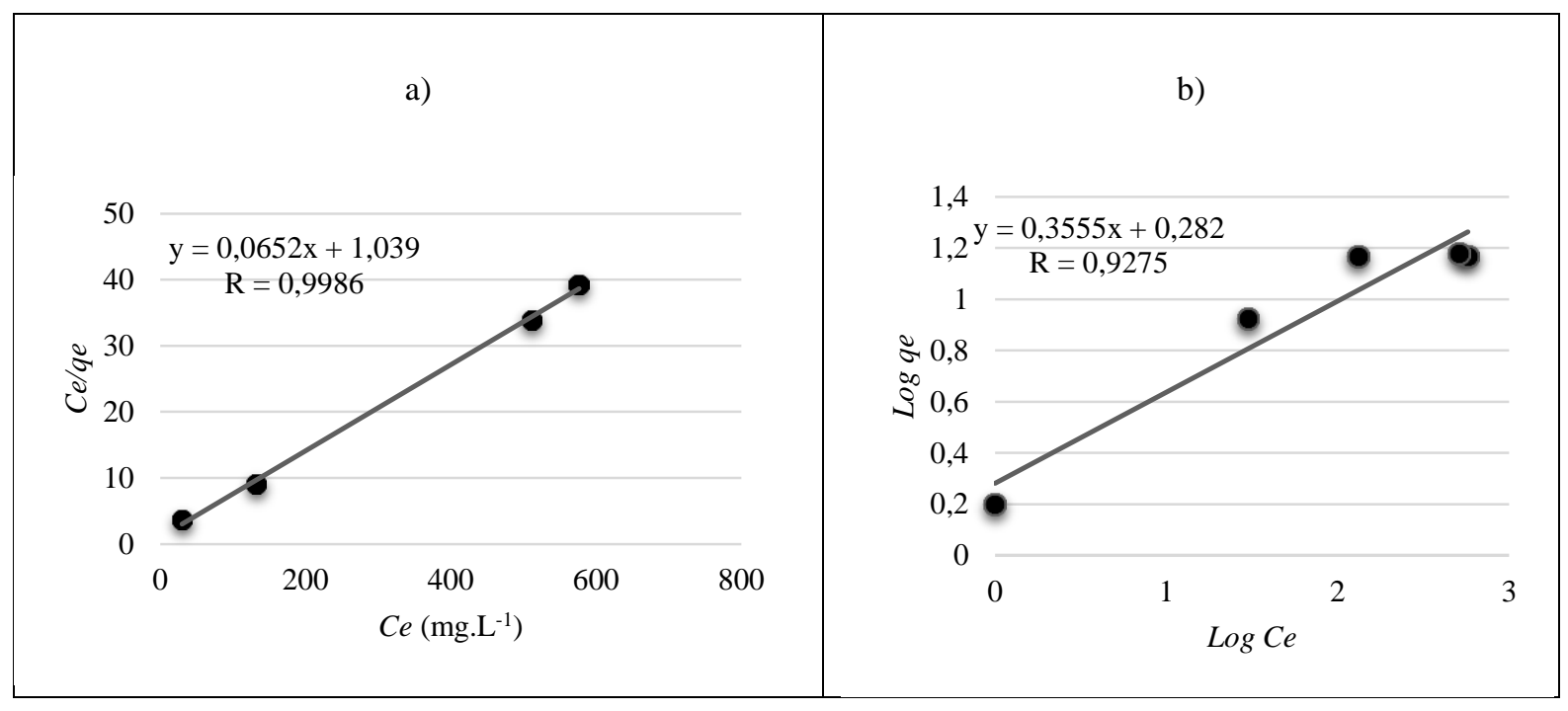

Figura 8: Forma linear das isoterma a) Langmuir e b) Freudlich para a adsorção de $\mathrm{Cu}$ (II) pelo bioadsorvente FBN.

Os parâmetros obtidos através do tratamento dos dados dos modelos de Langmuir e Freundlich estão representados na Tabela 1.

Tabela 1: Parâmetros dos dados tratados nos modelos lineares de Langmuir e Freundlich

\begin{tabular}{c|c|c|c|c|c|c|c}
\hline \multicolumn{8}{c}{ Parâmetros para o íon metálico Cu } \\
\hline \multicolumn{4}{c}{ Modelo Langmuir } & \multicolumn{4}{c}{ Modelo Freundlich } \\
\hline $\boldsymbol{q}_{\max }$ & $\boldsymbol{K L}$ & $\boldsymbol{R}$ & $\boldsymbol{R}$ & $\boldsymbol{n}$ & $\mathbf{1} \boldsymbol{n}$ & $\boldsymbol{K} \boldsymbol{F}$ & $\boldsymbol{R}$ \\
\hline 15,34 & 0,063 & 0,025 & 0,99 & 2,81 & 0,35 & 1,91 & 0,92 \\
\hline
\end{tabular}

Sendo assim, analisando os dados obtidos na Tabela 1 observa-se que os modelos matemáticos de Langmuir e Freudlich são capazes de descrever o comportamento do processo adsortivo de $\mathrm{Cu}$ (II) pelo adsorvente FCB. Pois, no modelo de Langmuir, a constante $K L$ com valor de 0,063 confirmou que houve uma adequada interação entre o adsorvato $\mathrm{Cu}$ (II) com o adsorvente FCB. Logo, o parâmetro de evolução de equilíbrio $R L$ de Langmuir com valor entre 0 e 1 representou uma excelente afinidade do adsorvato pela fase sólida à fase líquida e, do mesmo modo, o modelo de Freundlich apresentou o valor $n$ entre 1 e 10 remetendo os dados experimentais a uma adsorção favorável. Todavia, o modelo matemático de Langmuir mostrou ser capaz de descrever melhor a adsorção, pois gerou um coeficiente de correlação linear $R$ mais alto.

De acordo com a Figura 9, em concentrações baixas, as isotermas não lineares dos modelos experimentais, Langmuir e Freundlich são muito similares. Contudo em altas concentrações a isoterma do modelo de Freundlich se diverge, dessa maneira corrobora com Cooney [18] quando afirma que este modelo não se ajusta à estas condições. 


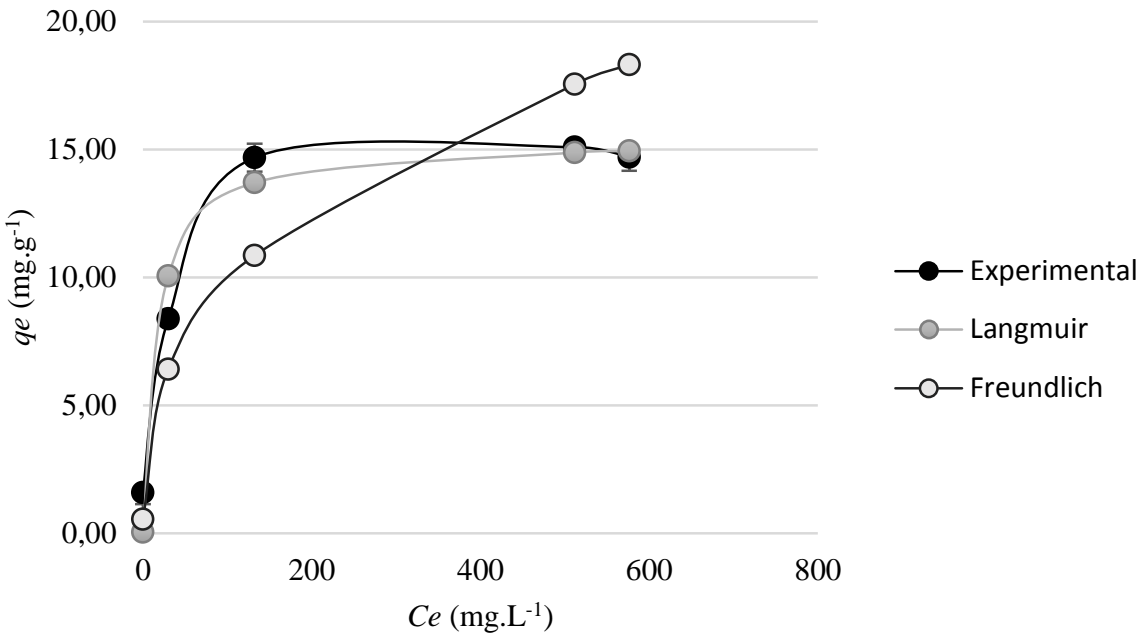

Figura 9: Capacidade adsortiva para os modelos estudados.

Cruz [5] e Nascimento et al [13] sugerem que o $q_{\max }$ obtido através do modelo de Langmuir representa a capacidade máxima de preenchimento dos sítios ativos do adsorvente pelo adsorvato. Neste trabalho $q_{\max }$ alcançou a $15,3 \mathrm{mg} \mathrm{g}^{-1}$, e a capacidade adsortiva dos dados experimentais foi próximo de $15,1 \mathrm{mg} \mathrm{g}^{-1}$, alcançando um percentual de preenchimento dos sítios na faixa dos 98,7\% para as melhores condições.

\section{CONCLUSÃO}

Diante dos resultados obtidos neste trabalho, pode-se concluir que a FCB é um bioadsorvente promissor para remediação de íons cobre em meio aquoso. A metodologia aplicada no pré-tratamento se demonstrou adequada para a obtenção e regeneração dos sítios ativos do bioadsorvente. A caracterização por MEV-EDX confirmou uma estrutura superficial compatível, bem como sua composição química antes e após o processo de adsorção, evidenciando a presença de íons Cu (II) no bioadsorvente após a adsorção. O método do FT-IR confirmou a existência de grupos funcionais responsáveis pela composição da casca de banana e formação sítios ativos como o grupo $\mathrm{OH}$ existentes em abundância em materiais lignocelulósicos responsáveis pela interação por coordenação com metais. De acordo com os estudos adsortivos, foi possível determinar o tempo de adsorção dos sítios ativos da FCB em 10 minutos, a melhor faixa de pH em 5 e a concentração máxima de saturação de $15,1 \mathrm{mg}$ de $\mathrm{Cu}$ (II)/g de FCB. O estudo da variação da temperatura apresentou uma influência no processo adsortivo, sugerindo características de interação física, visto que $\mathrm{q}_{\mathrm{e}}$ decai com o aumento da temperatura. E ainda, a adsorção em monocamada confirmada pelo modelo de Langmuir, o qual se ajustou melhor ao comportamento do sistema de adsorção do íon cobre. Finalmente, mediante o interesse em adquirir um material alternativo que seja de baixo custo, abundante, renovável e que possua potencial para tratamento de efluentes contendo cobre, o bioadsorvente de FCB se mostrou eficaz devido à alta capacidade de adsorção, pois com $1 \mathrm{~kg}$ de FCB pode-se adsorver, aproximadamente, 15,1 g de Cobre nas condições apropriadas. Portanto, o estudo concluiu que a FCB é uma alternativa favorável e economicamente viável para remoção de cobre em meio aquoso.

\section{BIBLIOGRAFIA}

[1] HOSSAIN, M. A., HAO NGO, H., GUO, W. S., et al., "Removal of copper from water by adsorption onto banana peel as bioadsorbent", International Journal of Geomate, v. 2, n. 2, pp. 227-234, June. 2012.

[2] VAGHETTI, J. C. P., "Utilização de biossorvente para remediação de efluentes aquosos contaminados com íons metálicos", Tese de D.Sc., Universidade Federal do Rio Grande do Sul, Porto Alegre-RS, 2009.

[3] ANNADURAI, G., JUANG, R. S., LEE, D. J., "Adsorption of heavy metals from water using banana and orange pells”, Water Science and Technology, v. 47, n. 1, pp. 185-190, Jan. 2003.

[4] SILVA, G.S., NETO, A. C., CAPRI, M. R., "Biossorção de Cr (VI) pela casca de banana nanica no tratamento de efluente", In: II Congresso Internacional - RESAG - EEL-USP, Lorena, Brasil, 2015. 
[5] CRUZ, M.A.R.F., "Utilização da casca de banana como biossorvente", Dissertação de M.Sc., UEL, Londrina-PR, 2009.

[6] SOUSA, F. W., MOREIRA, S.A., OLIVEIRA, A. G., et al., "Uso da casca de coco verde como adsorvente na remoção de metais tóxicos”, Química Nova, v. 30, n. 5, pp. 1153-1157, Jul. 2007.

[7] SILVA, N. C. R. "Utilização da casca de banana como biossorvente para a adsorção de chumbo (ii) em solução aquosa”, Dissertação de M.Sc., Universidade Tecnológica Federal do Paraná, Campo Mourão, 2014.

[8] RODRIGUES, R. F., TREVENZOLI, R. L., SANTOS, L. R. G., et al., "Adsorção de metais pesados em serragem de madeira tratada com ácido cítrico", Engenharia Sanitária Ambiental, Rio de Janeiro, v. 11, n.1, pp. $1413-1452,2006$.

[9] CARVALHO, L. R., OLIVEIRA, E. A., KAMIMURA, E. S., et al., "Bioadsorção de crômio (VI) em casca de banana nanica (Musa paradisíaca L.) em pó em frascos agitados e em leito fixo", In: XVI Congresso Brasileiro de Engenharia Química - EMBEC - Fortaleza/CE, 2016.

[10] MARTINS, W. A., OLIVEIRA, A. M. B. M., MORAIS, C. E. P., et al., "Reaproveitamento de resíduos agroindustriais de casca banana para tratamento de efluentes", Revista Verde de Agroecologia e Desenvolvimento Sustentável, Pombal - PB - Brasil, v. 10, n.1, pp. 96-102, 2015.

[11] CETESB, Ficha de Informação Toxicológica - Cobre, In: Divisão de toxicologia, genotoxicidade e microbiologia ambiental, Brasil, 2012.

[12] CONAMA. MINISTÉRIO DO MEIO AMBIENTE. Resolução n430, de 13 de maio de 2011. Dispõe sobre as condições e padrões de lançamento de efluentes, complementa e altera a resolução $n^{\circ} 357$, de 17 de março de 2005, do Conselho Nacional do Meio Ambiente - CONAMA. Brasília, DF, 1991. Disponível em: <http://www.mma.gov.br/port/ conama/legiabre.cfm?codlegi=646>. Acessado em: 22 jan. 2018.

[13] NASCIMENTO, R.F., LIMA, A.C.A, VIDAL, C.B., et al., Adsorção: Aspectos Teóricos e Aplicações Ambientais, Fortaleza, Imprensa Universitária, 2014.

[14] ALI, R. K.., HULYA, K., "Adsorption of $\mathrm{Pb}$ (II) ions from aqueous solution by native and activated bentonite: kinectic, equilibrium and thermodynamic study", Journal of Hazardous Materials, v. 179, n. 1-3, pp. 332-339, 2010.

[15] SOUSA NETO, V. O., OLIVEIRA, A.G., TEIXEIRA, R. N. P., et al., "Use of coconut bagasse as alternative adsorbent for separation of copper (ii) ions from aqueous solutions: isotherms, kinetics, and thermodynamic studies", Bioresources and Technology, v. 6, n. 3, pp. 3376-3395, 2011.

[16] LANGMUIR, I., "The adsorption of gases on plane surfaces of glass, mica and platinum", Journal of American Chemical Society, v. 40, n. 9, pp. 1361-1403, 1918.

[17] MCKAY, G., BLAIR, H.S., GARDNER, J.R. "Adsorption of dyes on chitin: equilibrium studies", Journal of Applied Polymer Science, v. 27, pp. 3043-3057, 1982.

[18] COONEY, D. O. Adsorption Design for Wastewater Treatment, Florida, CRC Press, 1999.

[19] FEBRIANTO, J., KOSASIH, A. N., SUNARSO, J., et al., "Equilibrium and kinetic studies in adsorption of heavy metals using biosorbent: a summary of recent studies", Journal of Hazardous Materials, v. 162, pp. 616-645, 2009.

[20] THIRUMAVALAVAN, M., "Transform infrared spectroscopic analysis of fruit peels before and after the adsorption of heavy metal ions from aqueous solution", Journal of Chemical \& Engineering Data, v. 56, n. 5, pp. 2249-2255, June. 2011.

[21] CHEN, W., XU, Y., HWANG W., et al., "Pretreatment of rice straw using an extrusion/extraction process at bench-scale for producing cellulosic ethanol", Bioresource Technology, v. 102, n. 22, pp. 1045110458, Nov. 2011.

[22] MARTÍN, C., ALMAZÁN, O., MARCET, M., et al., "A study of three strategies for improving the fermentability of sugarcane bagasse hydrolysates for fuel ethanol production", International Sugar Journal, Glamorgan, v. 109, n. 1267, pp. 33-39, Jan. 2007.

[23] SOUZA, J. L., "Mesocarpo do coco verde (cocos nucífera) como adsorvente para os corantes: turquesa remazol e azul remazol”, Dissertação de M.Sc., UFMA, São Luís - MA, Brasil, 2009.

[24] MEMON, J. R., MEMON, S. Q., BHANGER, M.I., et al., "Characterization of banana peel by scanning eléctron microscopy and FT-IR spectroscopy and its use for cadmium removal", Colloids and Surfaces B: Biointerfaces, v. 66, n. 2, pp. 260-265, 2008. 
[25] CORTI. G.C., BOTARO, V.R., GIL, L.F., et al., "Estudo da capacidade de complexação de ions cu2+ em solução aquosa usando celulose modificada com anidrido succínico e com poliamidas", Polímeros: Ciência e Tecnologia. São Carlos- SP. v. 14, n. 5, pp. 313-317, 2004.

[26] TOLEDO, B. I. " Bisphenol a removal from water by activated carbon, effects of carbon characteristics and solution chemistry", Environmental Science Technology, v. 39, n. 16, pp. 6246-6250, 2005.

[27] NASCIMENTO, R.F., SOUZA NETO, V.O., MELO, D.Q. Uso de bioadsorventes lignocelulósicos na remoção de poluentes de efluentes aquosos, 1 ed., Fortaleza, Imprensa Universitária, 2014.

[28] ANNADURAI, G., LEE, D. J. "Equilibrium studies on the adsorption of acid dye into chitin", SpringerVerlag, v. 6, pp. 77-81, 2008.

\section{ORCID}

Emerson Leandro-Silva

Angelo Ricardo Favaro Pipi

Aroldo Geraldo Magdalena

Marina Piacenti-Silva https://orcid.org/0000-0001-6301-6075

https://orcid.org/0000-0003-1528-8724

https://orcid.org/0000-0003-3385-2106

https://orcid.org/0000-0001-7096-3652 